\title{
Improved kymography tools and its applications to mitosis
}

\begin{abstract}
António J. Pereira ${ }^{1}$ and Helder Maiato ${ }^{1,2}$
${ }^{1}$ Chromosome Instability \& Dynamics Laboratory, Instituto de Biologia Molecular e Celular, Universidade do Porto, Rua do Campo Alegre 823, 4150-180 Porto, Portugal

${ }^{2}$ Laboratory of Cell and Molecular Biology, Faculdade de Medicina, Universidade do Porto, 4200-319 Porto, Portugal
\end{abstract}

Keywords: kymograph, chromo-kymograph, guided-kymograph, chromo-projection, mitotic spindle

Corresponding author:

António J. Pereira

Instituto de Biologia Molecular e Celular

Rua do Campo Alegre 823

4150-180 Porto

Portugal

Tel: +351226074900

Fax: +351 226099157

e-mail: apereira@ibmc.up.pt 


\section{Abstract}

Although applicability of kymographs is limited to nearly one-dimensional (1D) processes, they have been instrumental in the analysis and interpretation of a wide range of dynamic biological processes. We focus here on some applications of kymography in the study of one among the range of 'nearly-1D' processes - mitosis. Using this biological context, we suggest generalized procedures in kymograph assembly that allow a partial retrieval of spatial information which is typically lost or distorted in conventional kymography. These kymograph variations, namely guided-kymography and chromo-kymography, are helpful in the determination of actual velocities and discrimination of structures when using thick regions of interest (ROIs). The method used to generate chromo-kymographs is generalized to other (non-kymograph) projection techniques, which include time-stack and z-stack projections.

\section{Introduction}

Analysis of biological processes generally involves the study of correlations amongst a large set of variables with the ultimate aim of extracting causal relations between them. Graphical representation of the dependencies between variables represents a crucial step towards true understanding of these problems. However, one often faces the problem of presenting multivariable data in the two-dimensional (2D) manuscript or computer monitor, a 'data compression' procedure which inevitably implies information loss. In fact, dynamic 2D representations in a computer and holography are not science fiction, but static 2D still is and will likely remain a compression procedure important for both visualization and comprehension of even the most complex data. We focus this article on a specific type of $2 \mathrm{D}$ graphical representation which is called a kymograph, useful in the study of dynamic 1D or quasi-1D processes. Mitosis, meiosis, 
neuron and filament-based protein traffic are among the range of quasi-1D processes amenable for kymograph analysis.

As a working frame, we will focus on the use of kymography to study mitotic spindle and chromosome dynamics. The ultimate goal of mitosis is to form two cells which are genetically identical to the precursor cell. To accomplish this, a microtubule-based spindle assembles around the chromatin after the nuclear envelope breaks down and attaches to all chromosomes through dedicated chromosome structures termed kinetochores (1). With the involvement of a large number of motor and non-motor proteins, the mitotic spindle is capable of driving chromosome alignment at the metaphase plate (2) and their subsequent segregation towards opposite sides of the cell. During mitotic progression, chromosome distribution evolves from a $3 D$ distribution inside the nucleus (at prophase) to a 2D distribution (at metaphase), thereby its name - metaphase plate. This remarkable conformational change is essential because it liberates one of the three spatial dimensions to define an axis for cell division. In fact, most chromosome and spindle internal dynamics occur preferentially along this axis. As an example, chromosomes typically display oscillations about the spindle equator during metaphase (3) and finally move processively towards opposite sides of the cell. Both movements are essentially parallel to the spindle axis, which in fact is the division axis. Also, microtubules are not static but undergo a permanent renewal process which involves their permanent poleward translocation, even while attached chromosomes remain still (or oscillating) at the metaphase plate $(4,5)$. Indeed, such poleward motion is also approximately parallel to the spindle axis and was termed microtubule poleward flux (4), a prominent process that occurs in most metazoans and plant spindles.

The above examples of quasi-linear motion are among the variety of mitotic processes amenable for kymograph analysis, of which we show some examples in this article. We will 
present an overview of conventional kymography and propose some improved variations and generalized procedures which help minimizing information loss. We conclude with a brief discussion on the use of those variations applied to time-projections. Admittedly, with one exception (the guided-kymograph, which we discuss as the first variation on kymography), the analysis tools presented here do not aim to (and do not) provide new quantification potential, but essentially assist visualization of multi-dimensional microscopy data in the 2D manuscript. All algorithms were implemented in Matlab programming environment (Matlab, MA; release 2007b).

\section{Conventional kymography}

The kymograph, popular among live cell microscopists, is a straightforward example of a compressed 2D representation. Kymography is useful when the configuration of the object of study evolves more slowly along one of the spatial dimensions than along the orthogonal dimension, allowing the 'slow' component to be considered frozen and disregarded. This liberates that spatial dimension to represent non-spatial variables, such as time. Originally, spatial assignment of time in this fashion was introduced by Carl Ludwig to graph fluctuations of a single variable over time (6). His device consisted of a pen whose vertical position responded to fluctuations of the monitored variable. The pen contacted with a record sheet which was translated by a drum revolving at constant velocity. In the context of live-cell microscopy, generation of a kymograph involves the extraction of a ROI from the acquired $2 \mathrm{D}$ images and the contiguous disposal of the obtained set of ROls along the axis of the 'non-informative' dimension (hereafter $y$-axis; see Fig.1). (Several freeware kymograph routines are available to generate kymographs, including ImageJ plugins. See for example at http://www.embl.de/eamnet/html/downloads.html). Fig. 1A-A' shows these steps applied to the 
analysis of a chromosome with fluorescently-labeled kinetochores moving towards the mitotic spindle equator. Note that after this operation the vertical axis of the kymograph (Fig.1 $\mathrm{A}^{\prime}$ ) is a spatial and a temporal axis at small and large scales, respectively. Although in this case we were able to display the kymograph without distorting each ROI image, it is frequent to have a much larger number of time frames or eventually thicker ROIs, a situation which precludes the undistorted visualization of each ROI image, unless a full kymograph with an unreasonable aspect ratio is, for some reason, acceptable. Given that in these cases information along the $y$ axis is effectively not visible, or maybe distorted by adjustment of the kymograph aspect ratio, it is often convenient to completely sacrifice the $y$ scale by performing an intensity projection along each column ( $y$ vector) of the ROI matrix. Examples of projected kymographs are shown in Figs. 1A" and 1B. Note that, after projection, the vertical axes become purely temporal: the two kymograph axes have now different units, allowing the aspect ratio of the kymograph to be adjusted at will. The details of the projection along the ROI columns are rather arbitrary (just as they are for z-projections, when intensity quantification is not an issue): maximal projection typically provides the sharpest kymographs but may be too selective if multiple structures of interest share an $x$-region, as often happens in thick-ROI kymography.

Kymographs are space-time descriptions and therefore represent a convenient means for measuring velocities. Velocity along $x, V_{x}$, is simply given by the slope of the track of interest in the kymograph relative to the temporal axis (Fig.1A"), while any residual $V_{y}$ component is effectively lost. How different is $V_{x}$ from the real velocity, $V$, depends on the angle $\theta$ between $\hat{x}$ and $\vec{V}$, and is given by $V_{x} / V=\cos (\theta)$, always less than one, i.e., the velocity measured through kymograph analysis is always an underestimation of the velocity, $V$, although fairly accurate if the structure of interest moves essentially along the $x$-axis. It may be useful to keep 
in mind that a process involving motion at less than $10^{\circ}$ relative to the $x$-axis will underestimate velocity with an error below $3 \%$.

The velocity derived from the slope of the kymograph track is the velocity of the object of interest (object $A$ ) relative to the lab frame. However, the biologically relevant velocity is probably one relative to some specific reference (object $B$ ), which is likely to be also moving relative to the lab frame. These two velocities (relative to the lab), $V_{A, l a b}$ and $V_{B, l a b}$, are calculated from the slope of the corresponding kymograph tracks, as discussed above. To calculate the velocity of object $\mathrm{A}$ relative to object $\mathrm{B}, V_{A, B}$, the classical rule for the addition of velocities is used, $V_{A, B}=V_{A, Z}+V_{Z, B}$. This is easy to understand if we think that the velocity we seek ( $A$ relative to $B$ ) is being calculated using an intermediary reference frame $Z$. In our case, $Z$ is the lab. The first term, $V_{A, l a b}$, is known from the track's slope in the kymograph, while the second term, $V_{l a b, B}$, is the negative of $V_{B, l a b}$ (the measured slope for object B). We emphasize that all velocities here mentioned are velocities projected along the $x$-axis, given that information on $V_{y}$ has been lost. To minimize the error associated with this incomplete information on velocity it may be convenient to choose a very thin ROI to assemble the kymograph, as this guarantees that any structure that eventually remains inside the ROI is migrating along the $x$ axis. The key problem here is that the structure of interest will very unlikely remain inside a very thin ROI. To be sure, this would be solved by choosing a thick ROI, which is exactly what we do not want to if velocity is to be calculated with confidence. We next describe a simple way to tackle the two problems (permanence time and velocity calculation).

\section{Guided-kymography}


In conventional kymographs, a single ROI is used to extract information from all sequential images, which leads to the abovementioned problems of i) guaranteeing the permanence of the object inside the ROI, especially if this is too thin and ii) allowing the correct estimation of velocity, which may become more critical when trying to solve the first problem. An easy way to circumvent these issues involves the use of a dynamic ROI, i.e., a ROI which is translated and rotated every frame to both capture the objects of interest and align the object velocity vector (relative to some meaningful reference) with the ROI $x$-axis. We called the output a 'guidedkymograph', which we describe next.

As a working frame, we will look at the example of measuring the velocity at which two kinetochores separate as anaphase progresses. The first step for generating the guidedkymograph involves tracking the two objects over time to generate two tables of $(x, y, t)$ coordinates, one for each object. For this step, we use in your lab a custom routine which determines the centroid of the object intensity distribution and searches for the next-frame centroid within a user-defined neighborhood. (This step is eventually preceded by 'weak' convolution to suppress noise at the pixel scale, background subtraction, or other preprocessing procedures that may increase the centroid positioning accuracy.) Of course, more sophisticated tracking routines are available (e.g., IDL, RyTrack) that may be used to generate and export the $(x, y, t)$ tables to the guided-kymograph generating routine. The second and last step involves the definition of the ROI side dimensions, not its position or orientation, as these will be automatically updated every time frame in accordance to the previously obtained tracking coordinates (Fig. 2A-C). The kymograph generated with such dynamic ROI now encloses the two references at all times and these have a relative velocity which is parallel to the $x$-axis of the kymograph. This means that slope calculations do not lead to underestimation of the relative velocity anymore. Depending on the exact purpose of the kymograph, it may be convenient to 
position the ROI so that the two structures are centered in it, or so that one of them remains in a static position relative to the dynamic ROI (Fig. 2D).

In what concerns velocity estimation, the $(x, y, t)$ tables already contain all the information needed, but there are several reasons to keep going and generate a (guided-)kymograph. One is that we biologists prefer images or other $2 \mathrm{D}$ representations to numbers emanating from computer programs. There is also an important reason, which is the fact that the tracked objects are often just reference points which define the main axis of the process but are not the object of interest themselves. As an example, we will measure the velocity at which microtubules translocate away from kinetochores in a metaphase-anaphase spindle. This is often an important quantity for researchers involved in the study of, for example, microtubule-kinetochore interactions, microtubule poleward flux or spindle force generation. To perform such measurements it is essential to mark the position of a reference portion of the microtubule fiber which is attached to the kinetochore. This is typically done by optical marking (point-bleaching (7) or point-activation $(4,8)$ ) or sparse stochastic labeling of tubulin molecules $(9)$, the latter being the technique used in our experiments. The frame of reference for the measurement of velocity is the kinetochore attached to the fiber, which in our cells is labeled with mCherry-CID (10). For this purpose, a ROI enclosing the microtubule fiber and the attached kinetochore could in principle be used to generate a kymograph, where the fiber translocation velocity could be calculated. However, the spindle often translocates and rotates during the observation period, therefore the need for a guided-kymograph. To do that, we first have to define two convenient reference points to guide the ROI. These could be for example the kinetochore and the pole which are interacting with the two ends of the microtubule fiber of interest; alternatively, the two kinetochores of the chromosome may be used as guiding points if the attached fibers are not too tilted relative to the inter-kinetochore axis. After tracking the reference points, their coordinates are used to guide the ROI, generating a kymograph which includes the tracks of 
interest (microtubule marks) along with the tracks of the reference objects. An example of such procedure is shown in Fig.2E, where two sister kinetochores were tracked from metaphase until the end of anaphase. We chose in this case to force the left kinetochore to be static relative to the ROI $\left(\Delta_{\text {particle }}=\right.$ constant $)$, which facilitates the observation of the switch from polymerization (outward translocation) to depolymerization (inward translocation or 'pacman') (11) at the microtubule-kinetochore interface as the cell enters anaphase.

It is important to clarify that the discussion on relative velocities (see 'Conventional kymography' above) is valid for guided-kymography also, with the subtle difference that the intermediate frame of reference now is not the lab but the ROI (in the conventional kymograph these were equivalent frames of reference, i.e., their relative velocity was zero). In practice, however, the steps to calculate relative velocities are exactly the same as before: slope measurement in the kymograph and subtraction.

\section{Chromo-kymography}

Conventional kymographs use three of the variables we are sensitive to - intensity, x-position and $y$-position. In the kymograph, these reflect three variables of the experiment - intensity, $x$ position and time. Information on $y$-axis has been lost in the process, but it may be partially rescued if we make use of yet another variable which the retina is sensitive to - color, therefore the name chromo-kymograph. This information rescue is particularly relevant when performing thick-ROI kymography, i.e., when multiple objects of interest span the $y$-axis, potentially sharing one region of the $x$-axis: the chromo-kymograph tries to improve the capability of discrimination of such structures that coalesce in the $x$ dimension but not in the $y$-dimension. The procedure can be implemented easily and follows along the lines outlined in Fig.3A. The essential step that 
differentiates a chromo-kymograph from a conventional kymograph lies in the way the ROI matrix is projected onto a vector. In the chromo-kymograph, instead of performing an average, maximum or other singular projection, the ROI matrix is decomposed into red-green-blue (RGB) color sheets in some way that forces objects with different $y$-positions to be differently distributed between the RGB sheets. Operationally, this amounts to multiplying (pixel-wise) the ROI matrix by R, G and B weight matrices (see Fig.3A). The essential and obvious advantage of this color-coding procedure is that, as opposed to conventional kymographs, it discriminates objects which, sharing the $x$-region, occupy different $y$-regions.

For several reasons, the particular shape to be used for the RGB filters is not obvious (see inset in Fig.3A). First, it is clear that the chromatic component of the kymograph is mainly useful to assist visualization and does not provide, in principle, an advantage in terms of quantitative information. (In any case, a more clear visualization will often mean a more correct assessment of the tracks to be quantified.) In this context, it would be sensible to use RGB filters that i) span the largest range of the visible spectrum and ii) compensate for our nonuniform sensitivity to different wavelengths. Although we did not go into these details, we tried some simple filter sets which, based on heuristic arguments, should harness color information with good efficiency. The simplest set is based on rectangular filters, which assign pure RGB colors to contiguous portions of the ROI. An object crossing the threshold $y$ coordinate will abruptly have its color changed. A smoother color assignment is obtained using linear or parabolic filters. While the former is no more than an arbitrary choice amongst 'smooth' functions, the latter has a deeper meaning, in the sense that it is a normalized set. Specifically, the three parabolas shown in Fig.3A guarantee that i) the red, green and blue components contribute equally to the kymograph (equal integrated weight), ii) their sum is 1 at every position along $y$ and iii) at $y=0,1 / 2$ and 1 the filter is pure blue, green and red, respectively. Some examples of chromo-kymographs are shown in Fig. 3B for synthesized and experimental data. 
The chromo version of the kymographs is most often useful and sometimes not useful. This, added to the simplicity of implementation and the high processing speed, makes it essentially unnecessary to decide if it is worthwhile to use chromo-kymographs or not. In our lab, we don't decide if 'in this case' we should generate a chromo-kymograph; we generate it, because it is as quick and because it is frequently more informative. Of course, amongst the set of filters implemented in our Matlab program, one is a set of unitary (non-selective) filters, whose application amounts to the particular case of generating a conventional (monochromatic) kymograph.

\section{Chromo-projections}

The principle applied to chromo-kymograph generation may be also applied to projections along dimensions other than $y$. For example, instead of sacrificing $y$-axis information, we may want to keep it while sacrificing (again, as little as possible) time information. Geometrically, what we do is to stack up all $2 \mathrm{D}$ images (time frames) to construct a $3 \mathrm{D}$ pixel matrix and perform a projection along the z-axis (time axis) of the 3D matrix. Conventional projection may be again based on some weighed combination of the intensity values along the column (maximum, average, standard deviation, etc.). However, when analyzing one chromatic channel of the acquisition only, it may be sensible to use color information to retrieve some information along the time-axis which was lost through conventional projection. As in the chromo-kymograph, we may perform a smooth chromatic distribution of the pixels lying along the pixel column. Doing this, we are doing a time-to-color assignment, instead of the $y$-to-color assignment used in the chromo-kymograph. For example, the RGB filters defined may be so that the first time frames are essentially red and the end of the experiment is essentially blue. Clearly, the only difference between chromo-projection and chromo-kymography lies in the geometrical definition of the 
columns that will be split into different chromatic components. In Fig.4 we show the chromoprojection of fluorescently-labeled kinetochores imaged from metaphase to anaphase. In any type of chromatic splitting (chromo-time-projection, chromo-kymograph, etc.) it may be useful to perform color-coding in only one region of the columns to be chromo-projected. As an example, we show in Fig.4C one such partial chromo-projection of the metaphase-anaphase kinetochores. As in the full chromo-projection case, coincident color means time coincidence, but here all chromatic information is condensed in the chosen time-window, which improves time resolution. This is a convenient means to visualize time-coincident events in the full-length spatial context of the process.

\section{Conclusion}

We presented some visualization procedures which may be useful for correct velocity quantification in kymograph analysis and for retrieval of information discarded in image projection procedures, of which the kymograph is one example. For correct velocity determination we focused on the generation of guided-kymographs, which rely on tracking of two reference objects that are used to define the ROI movement from frame to frame, thus compensating for rotation and translation of the structures of interest. In this way, we prevent the structure to leave the $\mathrm{ROI}$ area and guarantee that the orthogonal axes of the final kymograph represent indeed a biologically meaningful frame of reference to calculate velocities. We next presented an easy-to-implement procedure which may be applied whenever color is not being used and some dimension of the acquisition is compressed by projection. In these cases, an alternative projection step may be defined that splits the pixel vectors under projection onto the RGB space, instead of simply collapsing it onto a number (intensity). Chromoprojections are extremely light implementations in terms of processing requirements; therefore our feeling that, although the (new) color-coded information has a limited added potential, it 
should be implemented as a default procedure in all projection routines (kymograph, timeprojection, z-projection, etc.). The color-coded version of the projection will often not yield new information, but will make it more clearly visible and discernable within its context.

\section{Acknowledgements}

We thank Irina Matos for providing experimental data for kymograph generation. A.J.P. was supported by Crioestaminal/Viver a Ciência and Fundação para a Ciência e a Tecnologia (FCT). Work in the lab. of H.M. is supported by grants PTDC/BIA-BCM/66106/2006 and PTDC/SAU-OBD/66113/2006 from FCT, and the Gulbenkian Programmes for Research Stimulation and Frontiers in the Life Sciences.

\section{References}

1. Walczak, C. E., and Heald, R. (2008) Int Rev Cytol 265, 111-58.

2. Kapoor, T. M., and Compton, D. A. (2002) J Cell Biol 157, 551-6.

3. Skibbens, R. V., Skeen, V. P., and Salmon, E. D. (1993) J Cell Biol 122, 859-75.

4. Mitchison, T. J. (1989) J Cell Biol 109, 637-52.

5. Zhai, Y., Kronebusch, P. J., and Borisy, G. G. (1995) J Cell Biol 131, 721-34.

6. Zimmer, H. G. (1996) Pflugers Arch 432, R9-22.

7. Buster, D. W., Zhang, D., and Sharp, D. J. (2007) Mol Biol Cell 18, 3094-104.

8. Ganem, N. J., Upton, K., and Compton, D. A. (2005) Curr Biol 15, 1827-32.

9. Danuser, G., and Waterman-Storer, C. M. (2006) Annu Rev Biophys Biomol Struct 35, 361-87.

10. Lince-Faria, M., Maffini, S., Orr, B., Ding, Y., Claudia, F., Sunkel, C. E., Tavares, A., Johansen, J., Johansen, K. M., and Maiato, H. (2009) J Cell Biol 184, 647-57.

11. Gorbsky, G. J., Sammak, P. J., and Borisy, G. G. (1987) J Cell Biol 104, 9-18. 


\section{Figure legends}

Fig.1. Kymograph generation. A) Kinetochore imaging during metaphase plate formation. The $\mathrm{ROI}$ defines the image window to be extracted for kymograph generation. $A^{\prime}$ ) The same ROI is extracted from all sequential images, which are stacked up along the $y$ dimension. In this configuration, the $y$-axis of the kymograph is both spatial and temporal. A") Projected kymograph from A', where each pixel column in each ROI is replaced by a single representative pixel value (in this case, the maximum pixel value). Average velocity between $t_{1}$ and $t_{2}$ corresponds to the slope of the line connecting the track at these time-points (short-dashed line). Instantaneous velocity is the slope of the line tangent to the track at that time-point (longdashed line). B) Example of a projected kymograph derived from metaphase-anaphase sequential imaging of kinetochores. In this case, the defined ROI encloses the whole set of kinetochores.

Fig.2. Guided-kymograph. A) Schematic representation of the guided-kymograph technique. A dynamic $\mathrm{ROI}$ undergoes translation and rotation according to the previously obtained tracking coordinates of 2 references. B) Example of the reference paths in the case of two anaphase kinetochores and display of the first and last kymograph ROls. C) Time-projection of the kinetochore pair paths inside the dynamic ROI. D) Two possible outcomes for the guidedkymograph. ROI centered on both references $\left(\Delta_{\text {center }}=\right.$ constant $)$ and ROI static relative to one of the references $\left(\Delta_{\text {particle }}=\right.$ constant $)$. E) Example of the usage of a guided-kymograph based on two references to observe the dynamics of a third element, which in this case is the microtubule fiber which emanates from the tracked sister-kinetochores. It is clearly visible that 
microtubules slip away from the kinetochore during metaphase (poleward flux) but switch to depolymerization ('pacman') at anaphase onset.

Fig.3. Chromo-kymograph. A) Schematic of the algorithm used to generate chromokymographs. The pixel matrix enclosed by the original ROI is (pixel-wise) multiplied by three $y$ dependent functions ( $R, G$ and $B$ spatial filters). These operations assign different spatial $y$ portions of the original ROI to different RGB components. The filter shapes (of which some examples are shown in the inset) determine the specific position-dependent color assignment. Superposition of the three RGB components defines a 'color' matrix with dimensions $n \times m \times 3$, where $n$ is the number of rows and $m$ is the number of columns of the matrix. Matrix projection along $y$ generates a 'colored' vector. The color of a specific pixel in the vector reflects the combined weights of the RGB filters weighed by the pixel values on the corresponding column of the original ROI. We call this a 'chromo-kymograph elementary vector' as opposed to the (conventional) 'kymograph elementary vector' which results from direct projection of the column elements. In both cases, the details of the projection are modulated by the specific type of projection used (maximum, average, etc). (B) Examples of chromo-kymographs generated from synthesized and experimental data. The synthesized data simulates anti-parallel particle flow along the $x$-axis with the two streams occupying different $y$-positions. In this case, the conventional kymograph does not allow vertical discrimination of the two flows, while observing the chromo-kymograph one could infer that the two flows do not overlap in space. Experimental data chromo-kymographs show (speckled) microtubules undergoing poleward flux in a metaphase anaphase cell (left) and kinetochore (middle) and chromosome (right) alignment and segregation. 
Fig. 4. Chromo-projection along time dimension. Projection of a time-lapse movie of fluorescently-labeled kinetochores in metaphase and anaphase. In (A) no chromo-projection was used while in (B) the chromo-kymograph routine projects the time-vectors of each $(x, y)$ pixel position. In (C) the chromo-projection was assigned to a limited time-window of the experiment, allowing a fine time-discrimination of events. To illustrate this, the chosen timewindow coincides with oscillations on anaphase kinetochore movement (arrow-heads), which can now be clearly identified as simultaneous, a fact that could only be guessed when looking at the conventional projection. Scale bar, $2 \mu \mathrm{m}$. 


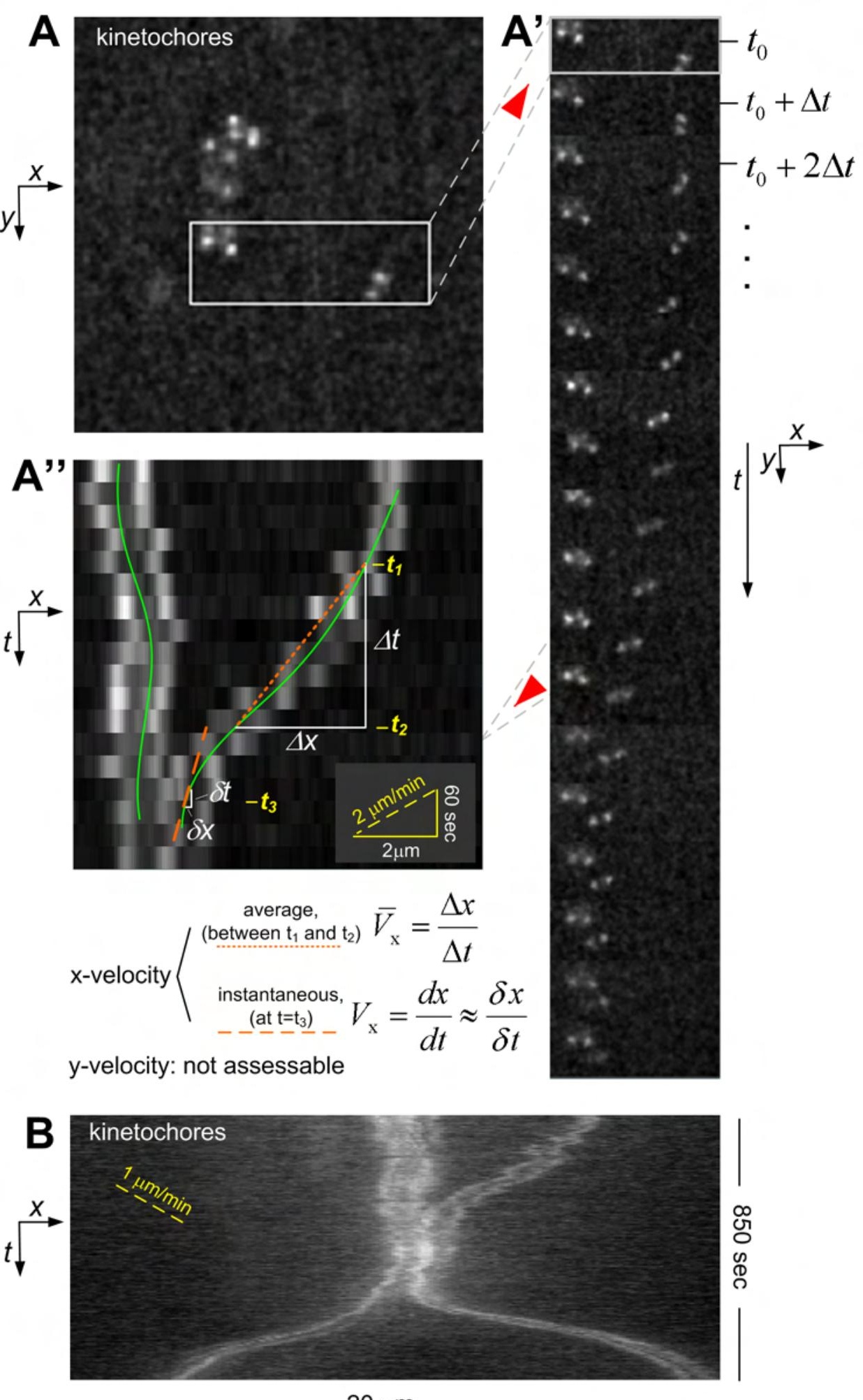


A $t_{0}$

\begin{tabular}{|l:l|}
\hline 0 & 0 \\
\hline
\end{tabular}

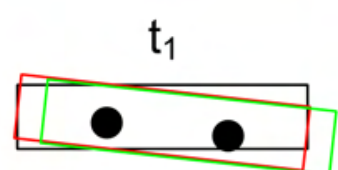

$\leftarrow \Delta_{\text {particle }}=$ constant

$\leftarrow \Delta_{\text {center }}=$ constant
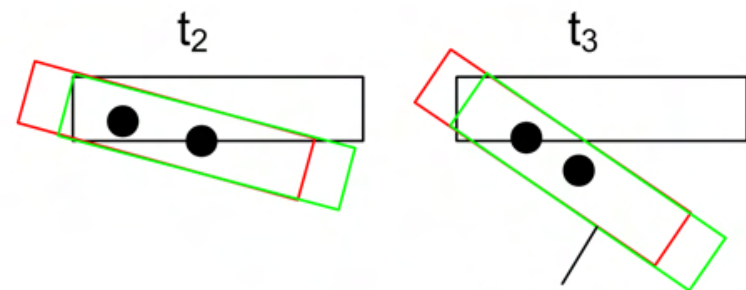

guided-kymograph

windows conventional

kymograph

window

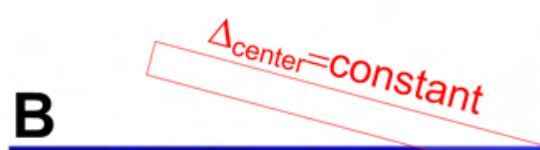

initial kinetochore positions

\section{kinetochores}

final leftkinetochore position

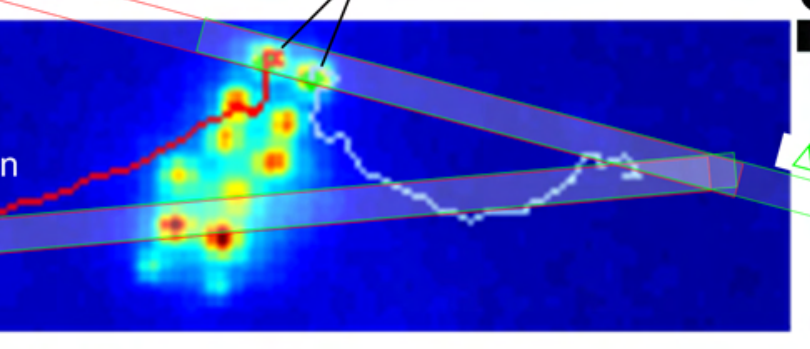

Kinetochores path C along dynamic $\mathrm{ROI}$

$\Delta_{\text {center }}=$ constant

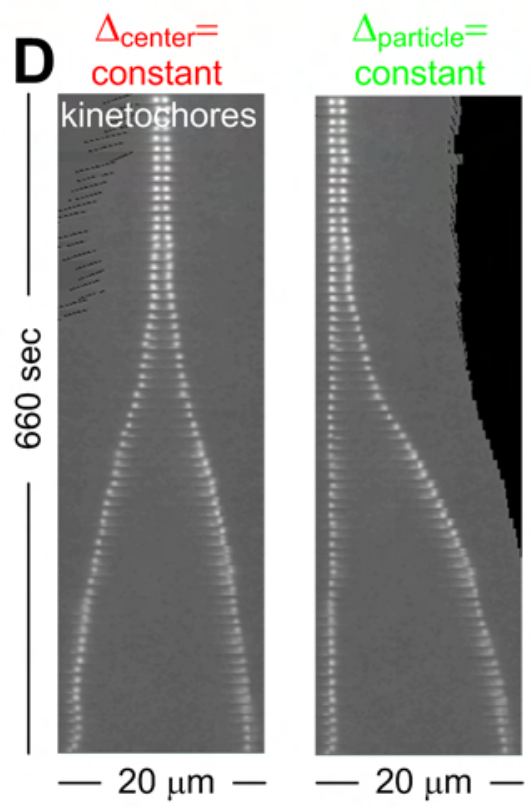

E

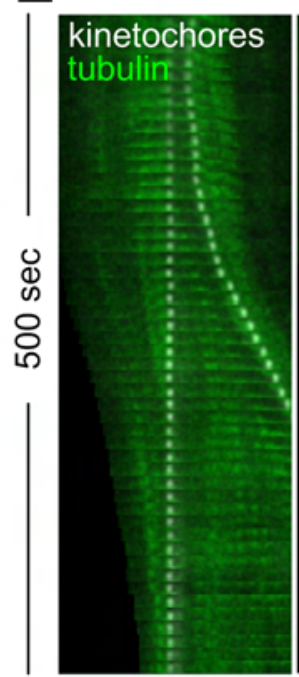

$14 \mu \mathrm{m}$
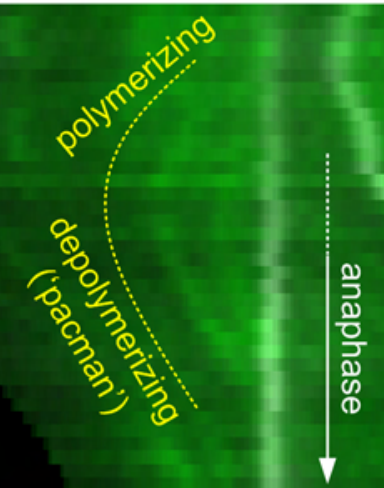

kinetochores tubulin

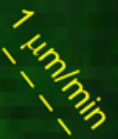


RGB spatial

filtered RGB components

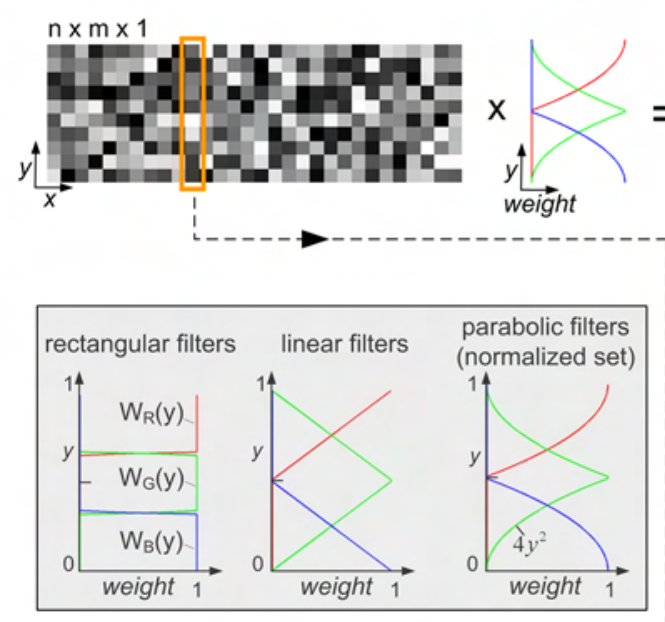

conventional kymograph projection

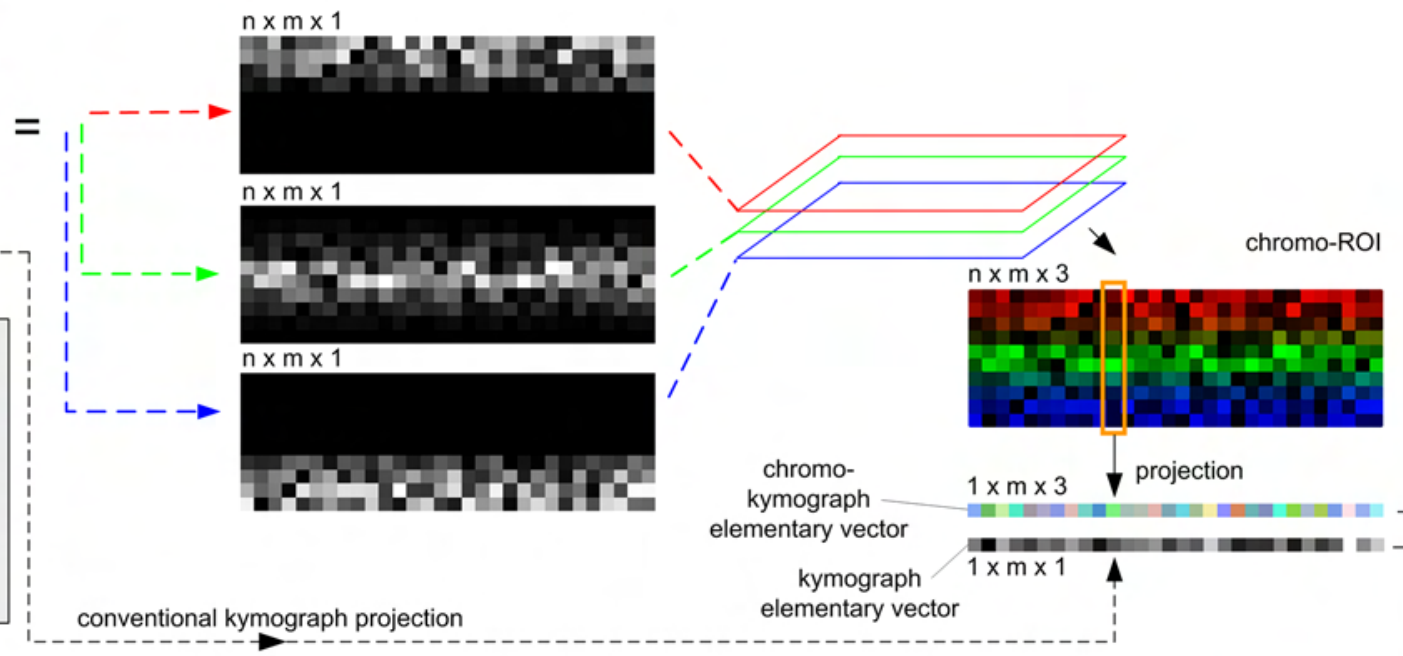

B

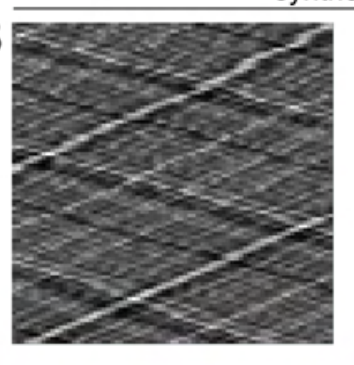

synthesized

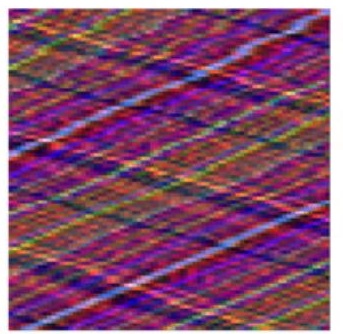

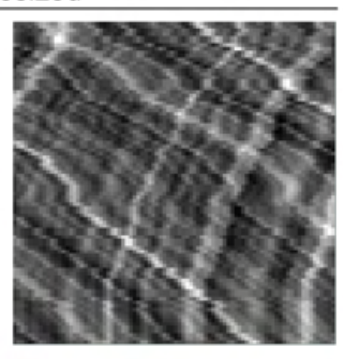

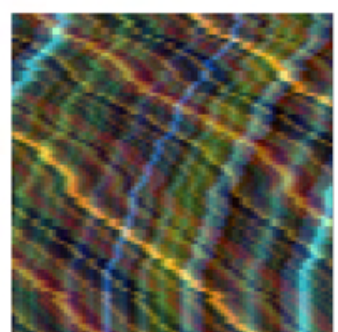

experimental
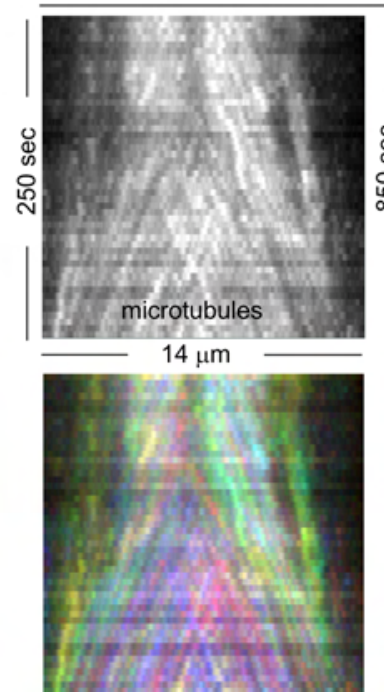

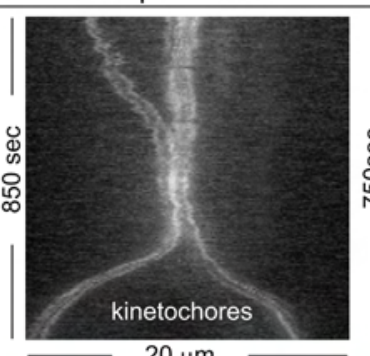

$20 \mu \mathrm{m}$

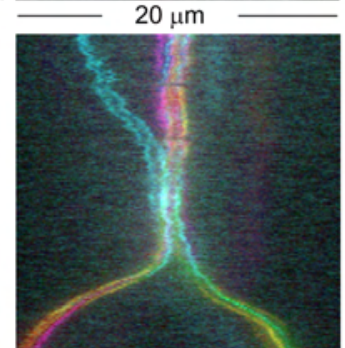

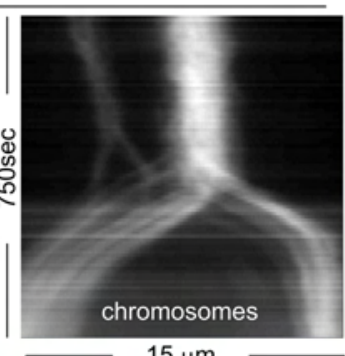

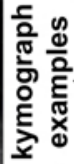

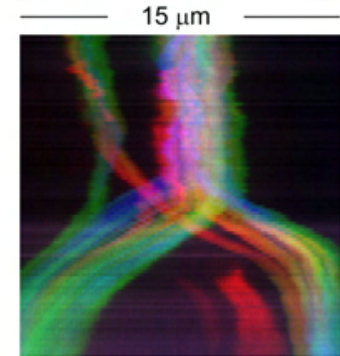

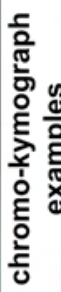




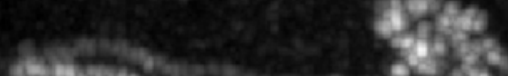

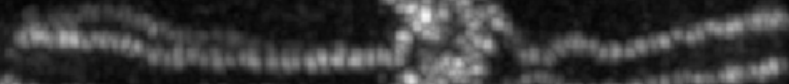

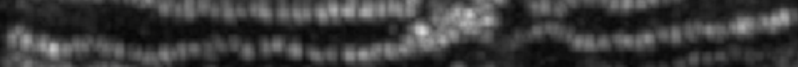

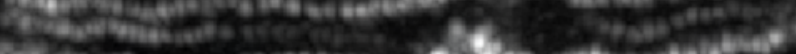

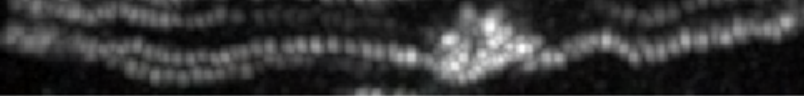
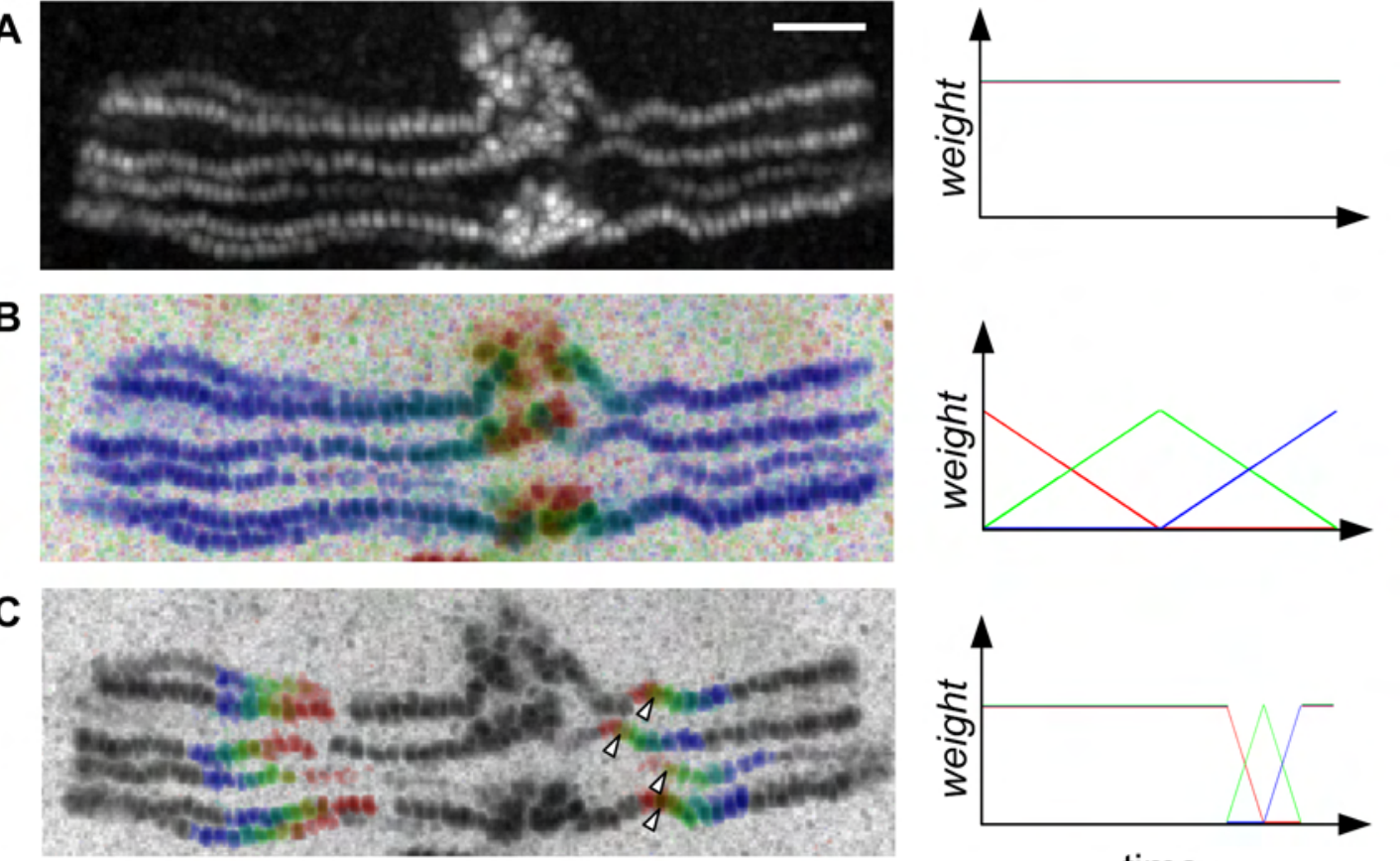

A
C

\section{B}

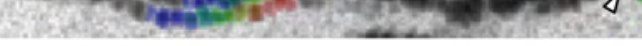
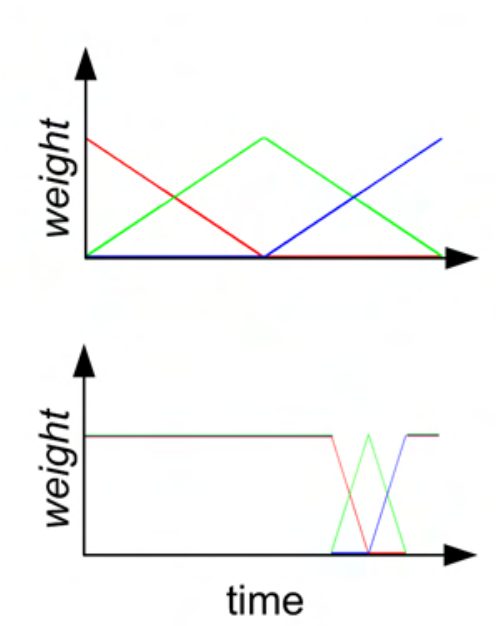

time 